Kapitel widmet sich daher explizit dem medialen Wandel als potentieller Prädiktor für das Aufkommen von Gegenöffentlichkeiten.

\title{
III.3 Medialer Wandel als Bedingung
}

Wie bereits in Kapitel II.3 ausgeführt, hat der mediale respektive digitale Wandel die öffentliche Kommunikation massgeblich transformiert. Dies trifft nicht nur auf die Öffentlichkeit zu, sondern auch gleichbedeutend auf Gegenöffentlichkeiten. Besonders Gegenöffentlichkeiten oder auch einzelne kritische Stimmen gegen die öffentliche Meinung profitierten vom Web 2.0 und der Möglichkeit, ohne grössere Barrieren Meinungen kundzutun wie auch selbst Informationen aufzubereiten und öffentlich zu verbreiten - sei es über Blogs oder Social-Media-Plattformen. Die Theoriefigur der Longtail-Öffentlichkeit wurde in diesem Kontext bereits ausführlich erläutert (Eisenegger, 2017, 2021; Neuberger, 2009) (vgl. Kapitel II.3.2). Grundgedanke dahinter ist - grob zusammengefasst -, dass am langen Schweif der Öffentlichkeit Lai_innen oder pseudowissenschaftliche Akteur_innen öffentlich kommunizieren - wenn auch mit geringeren Reichweiten im Vergleich zu den Massenmedien - und potentiell in Konkurrenz zum professionellen Informationsjournalismus treten. Auch Castells (2007) beschreibt dieses Phänomen und verwendet dabei den Begriff der Counter-power, der Gegenmacht. Auch Fenton und Downey (2003) verweisen in diesem Kontext auf Castells Konzept der Netzwerkgesellschaft, um Gegenöffentlichkeiten im Internetzeitalter einzuordnen. Die Autor_innen versuchen, das Öffentlichkeitskonzept von Habermas (2018 [1962]) unter Bezugnahme auf Castells (2010 [1996]) zu adaptieren, indem sie die Rolle von Gegenöffentlichkeiten verdeutlichen. Fenton und Downey (2003) sehen den Zuwachs an Gegenöffentlichkeiten in der dynamischen Beziehung zur herrschenden Öffentlichkeit begründet, die durch eine Krise geprägt sei. Ursächlich für die Krise der Öffentlichkeit respektive Demokratie sind nach den Autor_innen vor allem das sinkende Vertrauen in die hegemoniale Öffentlichkeit, Globalisierungstendenzen und der Neoliberalismus. Der Aufstieg von Gegenöffentlichkeiten (respektive eine zunehmende Solidarität von Teilöffentlichkeiten) in Netzwerkgesellschaften resultiert zudem daraus, dass der technologische Wandel eine Vernetzung von Akteur_innen über grosse geographische Distanzen ermöglicht - weswegen die Autor_innen vor allem die Globalisierung moderner Gesellschaften als zentrale Bedingung für die Etablierung von Gegenöffentlichkeiten erachten. In ähnlicher Weise argumentieren Pfetsch, Löblich und Eilders (2018), unter Verwendung des Begriffs «dissonante Öffentlichkeiten». Dissonanzen werden in diesem Kontext als ein Charakteristikum von Gegenöffentlichkeiten betrachtet, «als Ausdruck expliziter Gegenrede» (Pfetsch et al., 2018, S. 482). Einen Anstieg an Dissonanzen in der Öffentlichkeit sehen die 
Autorinnen in der digitalen Kommunikationsinfrastruktur begründet, im Sinne von Plattformen, Foren oder Netzwerken, auf denen neue Akteur_innen, abseits von professionellen Gatekeepern, eine Sprecherrolle einnehmen könnten. Digitale Öffentlichkeiten sind demnach von einer höheren Partizipationskraft, Austragung von Konflikten und Mobilisierungstendenzen geprägt. Für Gegenöffentlichkeiten erweist sich die Nutzung von beispielsweise Digitalplattformen als grosses Potential (Pfetsch et al., 2018). Schink (2020, S. 319), der Gegenöffentlichkeiten als «Kontraposition zu gesellschaftlich-etablierten Institutionen, «Eliten〉 sowie zum politisch-medialen 〈Mainstream»» definiert, betrachtet in ähnlicher Weise das Internet als «die Technologie der «Gegenöffentlichkeit [Hervorhebung im Original]»». Der Autor stellt in diesem Zusammenhang die relationale Perspektive zwischen Öffentlichkeit und Gegenöffentlichkeit heraus, wonach sich Gegenöffentlichkeit durch Referenzierung auf den so genannten Mainstream reproduziere. Auch Kaiser und Rauchfleisch (2019) betonen die Wichtigkeit von Social-Media-Plattformen für Gegenöffentlichkeiten, da hier neben der Möglichkeit der Vernetzung auch eine prinzipielle Freiheit der Meinungsäusserung gegeben sei. Die Autoren gehen davon aus, dass sich vor allem extreme Bewegungen, beispielsweise aus dem rechten politischen Lager, die Möglichkeiten von Social Media für die Vernetzung (im Sinne einer Gemeinschafts- und Identitätsformierung), Mobilisierung und Verbreitung ihrer Ansichten zu Nutze machen. Erst durch die Konstitution einer kollektiven Identität entsteht die Möglichkeit für Gegenöffentlichkeiten, die öffentliche Agenda zu erreichen respektive $\mathrm{zu}$ formieren. Sobald sie die öffentliche Agenda mitbestimmen, können sie folgend nicht mehr als Gegenöffentlichkeiten bezeichnet werden (Kaiser \& Rauchfleisch, 2019). Engesser und Wimmer (2009) heben in diesem Zusammenhang das Konzept des partizipativen Journalismus - oder Bürger_innenjournalismus respektive citizen journalism - als kennzeichnend für Gegenöffentlichkeiten im digitalen Zeitalter hevor. Partizipativer Journalismus nach Engesser (2008, S. 66) «beteiligt die Nutzer maßgeblich am Prozess der Inhaltsproduktion, wird außerhalb der Berufstätigkeit ausgeübt und ermöglicht die aktive Teilhabe an der Medienöffentlichkeit.» Der Autor unterscheidet zudem zwischen vier Formen des partizipativen Journalismus, darunter Weblogs, Kollektivformate, professionell-partizipative Nachrichtensites und Leserreporter-Rubriken (vgl. hierzu auch Engesser \& Wimmer, 2009). Relativ jung ist die Erforschung von professionell-partizipativen Nachrichtenseiten, bei denen Nutzer_innen Beiträge liefern, die allerdings von einer professionellen Redaktion bearbeitet werden (Engesser \& Wimmer, 2009). Auch partizipativer Journalismus hat eine längere Tradition - noch vor der Etablierung des Internets - z. B. in Form von Leserbriefen oder der bereits erwähnten Alternativpresse. Dem Potenzial der Online-Kommunikation wird in diesem Zusammenhang aber eine besondere Bedeutung zugemessen (Engesser, 2008). Die aktuelle Forschung fokussiert entsprechend vor allem Online-Tätigkeiten im Kontext von 
partizipativem Journalismus: «Citizen journalism» refers to a range of web-based practices whereby <ordinary» users engage in journalistic practices» (Goode, 2009, S. 1287). Praktiken von Lai_innen, wie Blogging, das Posten von Kommentaren auf Digitalplattformen oder das Teilen von Fotos und Videos, werden in diesem Zusammenhang herausgestrichen (Goode, 2009).

Engesser und Wimmer (2009) gehen folgend davon aus, dass Gegenöffentlichkeiten (oder: kritische Teilöffentlichkeiten) aktuell auf digitale Kanäle geradezu angewiesen sind, bzw. dass diese in besonderem Masse von der Konnektivität des Internets profitieren, wonach sich Akteur_innen einfacher miteinander vernetzen können. Partizipativer Journalismus im Internet nimmt weiter eine Korrektivfunktion gegenüber dem etablierten Journalismus ein, was auch in einer klaren Gegenthematisierung erfolgen kann - dies allerdings mit vergleichsweise geringen Reichweiten (Engesser \& Wimmer, 2009). Neuberger (2012) betont in diesem Zusammenhang eine zunehmende Vermischung von Bürger_innenjournalismus, professionellem Journalismus wie auch nichtjournalistischer Kommunikation auf Digitalplattformen. Journalismus durch Lai_innen impliziert weiter eine stark normative Komponente, im Sinne einer «Verbesserung der öffentlichen Kommunikation» durch deren Partizipation, der Erfüllung deliberativer Werte für den öffentlichen Diskurs und der Möglichkeit für Bürger_innen, eine journalistische Vermittlungsrolle einzunehmen (Neuberger, 2012, S. 55). Neuberger (2012) weist jedoch gleichzeitig darauf hin, dass an den partizipativen Bürger_innenjournalismus dieselben Qualitätskriterien wie an den professionellen Journalismus herangetragen werden müssten, da dieser nur in dieser Weise ein demokratisch-funktionales Äquivalent darstellen könne.

Eine durch den medialen Wandel bedingte Ausformierung von Gegenöffentlichkeiten zeigt sich anhand des Phänomens der Alternativmedien respektive alternativen Nachrichtenmedien. Das folgende Kapitel widmet sich zentral dem Untersuchungsgegenstand der vorliegenden Arbeit.

\section{III.4 Alternativmedien als Gegenöffentlichkeiten}

Die in diesem Hauptkapitel geschilderten Definitionen von Gegenöffentlichkeiten und sozialen Bedingungen für deren Entstehung verdeutlichen, dass diese in unterschiedlicher Weise auftreten können. So werden häufig soziale Bewegungen oder sonstige Protestbewegungen als Beispiele für «autonome Öffentlichkeiten» aufgeführt, die sich gegen die hegemoniale Öffentlichkeit positionieren. Mit einem Blick auf die neuen sozialen Bewegungen der 1960er und 1970er Jahre zeigt sich, dass auch diese Bewegungen mit Kommunikationsmitteln wie der «Alternativpresse» gearbeitet haben (z. B. Wimmer, 2015). Aber auch schon deutlich früher, in Zeiten der Französischen Revolution, lässt sich ein Aufschwung der 